Intertemporal choice reflects value comparison rather than self-control: insights from confidence judgments

Adam Bulley ${ }^{1,2 *}$, Karolina M. Lempert ${ }^{3}$, Colin Conwell ${ }^{1}$, Muireann Irish ${ }^{2}$, and Daniel L. Schacter ${ }^{1}$

${ }^{1}$ Department of Psychology, Harvard University, Cambridge, MA, USA

${ }^{2}$ The University of Sydney School of Psychology and Brain and Mind Centre, NSW, Australia

${ }^{3}$ Department of Psychology, University of Pennsylvania, Philadelphia, PA, USA

* Corresponding author

Keywords: self-control, intertemporal choice, decision-making, metacognition, confidence, value-based choice 


\begin{abstract}
Intertemporal decision-making has long been assumed to measure self-control, with prominent theories treating choices of smaller, sooner rewards as failed attempts to override immediate temptation. If this view is correct, people should be more confident in their intertemporal decisions when they "successfully" delay gratification than when they do not. In two preregistered experiments with built-in replication, adult participants $(n=117)$ made monetary intertemporal choices and rated their confidence in having made the right decisions. Contrary to assumptions of the self-control account, confidence was not higher when participants chose delayed rewards. Rather, participants were more confident in their decisions when possible rewards were further apart in time-discounted subjective value, closer to the present, and larger in magnitude. Demonstrating metacognitive insight, participants were more confident in decisions that better aligned with their independent valuation of possible rewards. Decisions made with less confidence were more prone to changes-of-mind and more susceptible to a patience-enhancing manipulation. Together, our results establish that confidence in intertemporal choice tracks uncertainty in estimating and comparing the value of possible rewards - just as it does in decisions unrelated to self-control. Our findings challenge selfcontrol views and instead cast intertemporal choice as a form of value-based decision-making about future possibilities.
\end{abstract}




\section{Intertemporal choice reflects value comparison rather than self-control: insights from confidence judgments}

Life is full of trade-offs between mutually exclusive possibilities, including those between smaller rewards available sooner, and larger rewards available after a delay. Choosing to delay gratification for larger, later rewards has long been viewed as the result of successful self-control, the ability to regulate impulses in the service of enduring goals (1). In laboratory intertemporal decision-making tasks, choices of larger, later reward have accordingly been interpreted as measuring a capacity for self-control, while opting for smaller, sooner rewards has been seen as a failure to overcome temptation $(2-8)^{1}$. This interpretation assumes that delaying gratification for long-term reward is the goal of participants in these tasks, and that participants are attempting to control themselves towards this end (8). One way to assess whether participants judge their choices as aligning with their goals is to gauge their degree of confidence in having made the right decisions. Here, we therefore ask whether a decisionmaker's confidence in making the "right" intertemporal choice reflects the extent to which that choice (a) objectively maximizes long-term reward, or (b) corresponds to their subjective evaluation of the options on offer. How do participants themselves judge whether the right intertemporal decision really is to delay gratification?

In domains from perception to memory, confidence in making the right decision tracks objective performance, albeit imperfectly (9-11). For instance, in a perceptual task such as judging whether a cloud of dots is moving mostly left or right, participants generally report higher confidence in their decisions when they achieve their goal of correctly identifying the direction of motion (12). Participants are also generally more confident in their decisions when the evidence more clearly favors one option - for instance because the motion is more obvious and easier to discriminate (13). Prominent frameworks therefore conceptualize confidence as the subjective belief that one's decision is correct, given the available evidence (14-16). In the context of intertemporal decision-making, if participants are attempting to execute self-control in pursuit of delayed gratification, we should expect them to be more confident in their decisions when they successfully achieve this aim. This assumption leads to a clear prediction from the view of intertemporal choice as a measure of self-control: participants should be more

\footnotetext{
${ }^{1}$ See supplementary material for representative examples of intertemporal decision-making tasks being interpreted as measuring a capacity for self-control.
} 
confident that they have made the right decision on average when they choose larger, later rewards.

An alternative view to the self-control account, however, leads to a different prediction. Instead of measuring a capacity for self-control per se, intertemporal choice can be conceptualized as a form of value-based decision-making, in which possible outcomes vary along multiple dimensions including their relative delays (17-21). With increasing delays, rewards tend to become less subjectively valuable, a phenomenon known as delay discounting. To determine this discounted value of delayed rewards, a decision-maker may sample evidence from memory in the service of prediction, for instance by anticipating the pleasure a larger, later payout might bring (22-27). Evidence accumulation is prone to noise and uncertainty, however, meaning that decisions do not always perfectly reflect the subjective value of available options. From this perspective, decision confidence would still track the belief in having made the correct decision (28), but in this case the correct decision is whichever option has the highest subjective value after taking into account their delays. A prediction from the view of intertemporal choice as value-based decision-making is therefore also clear: participants should be more confident that they have made the right decision when they choose whichever option has a higher time-discounted subjective value - regardless of whether that is the smaller, sooner reward or larger, later reward on offer.

Important recent work by Soutschek, Tobler, and colleagues $(29,30)$ supports the notion that participants reach greater confidence when their intertemporal decisions more closely reflect the time-discounted value of available options (as computed from those decisions). However, there is a problem that arises from measuring confidence in decisions and computing the value of the options from those very same decisions (see argument in 28). This circularity precludes knowing whether confidence reflects uncertainty in translating between each participant's own subjective evaluation of various options and their actual decisions about those options - as would be predicted by the value-based decision-making view. To decipher the role of intertemporal decision confidence, we need an independent index of the subjective, time-discounted value of available options.

Here, we designed a paradigm wherein participants first reported their confidence while making intertemporal decisions between monetary rewards available "today" versus after various delays, before they then separately estimated the time-discounted subjective value of the larger, later rewards in an independent bidding procedure (31). In this "Bidding task", participants completed fill-in-the-blank questions in the format: "I feel that receiving $\$ 25$ in 98 days would be about the same as receiving \$ today". By eliciting such an independent index 
of subjective value, our procedure enabled us to dissociate decision confidence from the timediscounted subjective value of the choice options, and thereby to systematically determine whether confidence in intertemporal choice follows the principles expected from either the self-control view or the value-based decision-making view. The latter would be supported if participants report higher confidence when their decisions line up more closely with their independent valuation of possible options. This would additionally constitute evidence of metacognitive insight, here quantified as the ability to know when one's actual decisions map on to one's subjective sense of what possible options are worth $(28,30)$.

In addition, we charted the specific question features that might affect confidence. While the magnitude of a reward and the delay to its receipt clearly have a synergistic effect on its subjective value, it is not clear how these attributes affect decision confidence. Recent modeling links both attributes directly to uncertainty in prospective value representations, suggestive of a potential role for confidence (32,33). Gabaix and Laibson (33) assume that the values of more distant rewards are estimated with greater uncertainty because simulations of the future become noisier with increasing temporal distance. In turn, Gershman \& Bhui (32) suggest that this uncertainty may be reduced by effortfully simulating the future with more precision when there is more reward on offer. Accordingly, if confidence tracks uncertainty in intertemporal value estimation, we should expect participants to be more confident in their decisions when available rewards are closer to the present and larger in magnitude.

Furthermore, we tested the hypothesis that low decision confidence would track choice inconsistency, as it does in other, non-intertemporal value-based decisions (34). We operationalized choice inconsistency both as deviations from a global computed preference function, and as changes of mind when we presented the same intertemporal choice question twice. Lastly, while intertemporal choices are highly malleable - influenced by contextual factors such as how a choice is framed (35) - the factors responsible remain poorly understood. One possibility is that decisions made with less confidence are more malleable, if indeed lower confidence indicates greater ambiguity in internal value evidence. Specifically, we tested a hypothesis that low decision confidence would predict subsequent susceptibility to the patience-enhancing effects of an explicit-zero framing manipulation, in which the opportunity costs of selecting an immediate reward are made salient $(36,37)$.

To presage the results, our investigation strongly suggests that confidence in intertemporal choice does not bear reliably on attempts to delay gratification, but instead follows the same principles as it does in other value-based decisions: tracking uncertainty in value estimation and comparison. The results thereby challenge an interpretation of delay 
discounting as a measure of attempted self-control and instead cast intertemporal choice as a form of value-based decision-making in which participants attempt to maximize timediscounted subjective value.

\section{Methods}

Participants made a series of choices between smaller, sooner (SS) amounts of money available "today", and larger, later (LL) amounts of money available at various delays in a standard "Monetary Choice Questionnaire" (MCQ) (38,39). LL reward amounts were always USD $\$ 25, \$ 30$, or $\$ 35$. SS rewards ranged from $\$ 10-\$ 34$, and delays ranged from 1-180 days. Participants encountered each MCQ question twice. In Experiment 1 (E1), trials from the two sets were intermixed randomly (but the same choice was never immediately repeated). In Experiment 2 (E2), participants made the second set of decisions separately, under explicit zero framing. For example, a standard question would be phrased as: "Would you prefer $\$ 18$ today or \$25 in 19 days?", while in the explicit zero framing phrased as: "Would you prefer $\$ 18$ today and $\$ 0$ in 19 days, or $\$ 0$ today and $\$ 25$ in 19 days?” Before the task, participants were told that on each day of data collection one participant would be selected and one of their choices would be paid out at the chosen time (and this condition was honored). After each decision in the MCQ, participants answered the following question on a 6-point scale from 1 (not at all confident) to 6 (absolutely certain): "How confident are you that the choice you made was the right one for you?". Upon finishing the MCQ and confidence portion of the experiment, participants completed the "Bidding task" based on Cooper et al., (31), with one bidding question matched to each of the 51 larger, later rewards from the MCQ (sliding scale from $\$ 0$ to $\left.\left[\mathrm{AMOUNT}_{\mathrm{LL}}\right]\right)$. We take responses to this Bidding task as an estimate of the value of each LL reward given its delay. See supplementary materials for more details on the tasks.

\section{Analysis of monetary choice questionnaire and Bidding data}

For each presentation of the MCQ choice set separately before any trial exclusions, individual participant data were fit with a logistic regression function using maximum likelihood estimation. The equation captures the probability of choosing the larger, later reward as a function of the difference in subjective value (SV) between the LL and SS reward.

$$
P_{L L}=\frac{1}{1+e^{-\beta\left(S V_{L L}-S V_{S S}\right)}}
$$


Where $P_{L L}$ is the probability of choosing the LL option, $S V_{L L}$ is the subjective value of the LL option, $S V_{\mathrm{SS}}$ is the subjective value of the SS option (assumed to be exactly the same as the objective SS amount, available "today"), and $\beta$ is a scaling factor that reflects the extent to which choices depend on the difference in subjective value between LL and SS options. The subjective value of the LL option was computed with a hyperbolic discounting function (40):

$$
S V_{L L}=\frac{A}{1+k D}
$$

Where $S V_{L L}$ is the subjective value of the LL option, $A$ is the objective amount of the LL option, $D$ is the delay to the LL option, and $k$ is a subject-specific free scaling parameter that reflects the effect of delay on $S V_{L L}$ (41). A higher $k$ value indicates that, for a given participant, rewards lose their subjective value more quickly with increasing delays to their receipt. In previous work, this hyperbolic discounting function well characterizes temporal discounting choice data $(38,42)$, as it did here (see supplementary analysis 1 ). To derive the subjective value difference between the options in a given choice (SVD), separately for MCQ subjective values (MCQ SV) and Bidding task subjective values (Bidding SV), we subtracted question specific $\mathrm{SV}_{\mathrm{SS}}$ (which we assume to be identical to the objective SS amount) from $\mathrm{SV}_{\mathrm{LL}}$, to compute a signed difference in subjective value between each option pair. Note that for both MCQ presentations, SVD is therefore the signed difference in subjective value between the two on-screen presented options. For the Bidding task presentation, SVD is the difference in value between self-reported $\mathrm{SV}_{\mathrm{LL}}$ and the (not presented) SS reward that was paired with that LL reward in the MCQ presentations. This is because no SS option was presented during the Bidding task.

\section{Participants}

The experimental protocol was approved by the Harvard University Institutional Review Board (protocol \#IRB20-0277). We aimed to collect 80 participants before exclusions in each experiment (pre-registered). A total of 81 participants completed E1 and 79 participants completed E2, after providing informed consent. Participants were Amazon mTurk users with a HIT approval rate $>90 \%$ located in the US and each received US\$5 for completing the study, which took 20 minutes. Experiments were administered using Qualtrics (Provo, UT). In E1, four participants were excluded for failing $>1$ attention check or missing an attention check question (detailed above), four were excluded due to a self-reported history of clinically 
diagnosed mental illness or neurological impairment, and two were excluded for providing no demographic data. Eight further participants were excluded for using only a single point on the confidence scale (all of whom used only the ceiling value of "absolutely certain"); this was a pre-registered criterion to bolster sensitivity in that key scale. After exclusions, our full E1 sample included 63 participants $(27 \mathrm{~F}, 36 \mathrm{M}$, aged 18-69, mean=37.68, SD=11.6). In E2, seven participants were excluded for failing $>1$ attention check, four were excluded due to a history of clinically diagnosed mental illness or neurological impairment, and seven were again excluded for using only a single point on the confidence scale (the ceiling value of "absolutely certain"). A further seven participants were excluded for having previously participated in one of our related mTurk studies (including E1). After exclusions, our full E2 sample included 54 participants $(23 \mathrm{~F}, 31 \mathrm{M}$, aged $24-60$, mean=34.94, $\mathrm{SD}=8.92)$. A small number of trials were removed from analyses in the following order: no choice data (E1: 75 trials, E2: 49), no confidence data (E1: 51 further trials, E2: 29), MCQ question time-out (E1: 19 further trials, E2: 19).

\section{Data analysis and models}

Data organization and plotting were performed in R-studio (v.3.6.2)(43) with the "Tidyverse" package (v.1.3.0)(44). Mixed effects models were computed using the "Ime4" package (v.1.1-23)(45) using a restricted maximum likelihood (REML) approach. The package "ImerTest" (v.3.1-2)(46) was used for estimating fixed effects $P$-values with Satterthwaite approximations. All predictors were z-scored across both MCQ presentations unless otherwise stated. The maximal starting mixed effects model structures allowed for random intercepts at the participant level, and random slopes by participant for all predictors. For further details on model specification see supplement section "model specifications". For models that had trouble converging as detailed in the supplement, we also ran Bayesian mixed effects regression versions, the results of which were consistent with those reported in the main text unless otherwise noted (supplementary material). 


\section{Results}

\section{Confidence tracks absolute difference in subjective value and varies with response time}

Before examining the role of confidence in intertemporal choice, we first established the SV of a given larger, later reward $\left(\mathrm{SV}_{\mathrm{LL}}\right)$ using two separate approaches. First, we used the pattern of MCQ choices separately in each MCQ presentation set to compute a participantspecific hyperbolic discounting function, and then used that function to compute the MCQ $S V_{L L}$ for each reward given its delay. Second, we defined $S V_{L L}$ from self-reported values in the Bidding task. We treat the response to the Bidding questions as the self-reported timediscounted Bidding $\mathrm{SV}_{\mathrm{LL}}$ for each LL reward. A mixed effects linear regression predicting MCQ $S_{L L}$ in the first choice presentation from Bidding $S_{L L}$ shows the significant relationship between these distinct estimates of subjective value, $\beta=0.72, t=12.90, P<$ 0.0001 , replicated in E2: $\beta=0.59, t=9.22, P<0.0001$. This finding suggests that subjective values computed with a hyperbolic model track closely with the independently self-reported subjective value of those LL rewards (see supplementary Fig.1).

In line with the value-based decision-making account, we hypothesized that participants would hold lower confidence in intertemporal decisions when options were more similar in subjective value, as defined by independent Bidding task self-reports. We also hypothesized that decisions closer to this indifference point would take longer because they require additional deliberation $(8,47-49)$, and that this effect would manifest as a negative relationship between response time and confidence $(8,22,42)$. In a mixed effects linear regression combining trials from the two MCQ presentations, the absolute difference in Bidding subjective value (Bidding-|SVD $\mid$ ) between the available choice options positively predicted MCQ decision confidence, in line with our pre-registered hypothesis, $\beta=0.13, t=$ 5.31, $P<0.0001$ (E2 Replication: $\beta=0.16, t=3.83, P<0.001$ ). In the same model, MCQ decision response time negatively predicted confidence, again in line with our pre-registered hypothesis $\beta=-0.23, t=-7.63, P<0.0001$, (E2 replication: $\beta=-0.16 t=-5.30, P<0.0001$ ). Note that Bidding- $|\mathrm{SVD}|$ was itself negatively correlated with MCQ decision response time as expected (across all trials, Pearson's $r=-0.17, P<0.0001,95 \%$ CI[-0.19, -0.14], (E2 replication: Pearson's $r=-0.15, P<0.0001,95 \% \mathrm{CI}[-0.17,-0.12])$. Together these results show that decisions made with less confidence were closer to independently self-reported subjective value indifference and took longer. 
Confidence is higher in decisions about rewards that are closer to the present or larger in magnitude

We hypothesized that participants would be more confident in their decisions when the LL option was closer to the present, given that simulating the future is a noisy process and may become progressively more noisy as the simulation time horizon increases, thereby increasing internal uncertainty around estimates of prospective value $(32,33)$. We found and replicated support for this pre-registered hypothesis: when LL rewards were closer to the present, decisions regarding those rewards were made with more confidence, $\beta=-0.08, t=-2.9, P=$ 0.0052, (E2 replication: $\beta=-0.06, t=-2.72, P=0.0089$ ). Furthermore, we found evidence to support another pre-registered hypothesis that when there was more money on offer participants would reach greater confidence, perhaps because they were investing more effort into making the right decision when more was at stake, $\beta=0.04, t=2.62, P=0.011$, (E2 replication: $\beta=0.08, t=3.78, P<0.001)$.

Note that because the variables covered in this section and the previous section were all included in the same mixed effects linear regression predicting confidence, all stated relationships hold when controlling for the other predictors (Fig.1.). While these pre-registered analyses treated confidence as a metric variable reflecting a continuous underlying judgement, we also ran sensitivity analyses using Bayesian mixed effects ordinal regression (50), and found the same pattern of effects (see supplementary material).

\section{Participants are not more confident when deciding to delay gratification}

In a mixed effects logistic regression predicting choices from confidence alongside the signed difference between participants' self-reported Bidding $S_{\text {LL }}$ and the amount of money offered "today" (Bidding-SVD), we found that participants were not more confident in decisions to delay gratification $\beta=-0.09, z=-0.8, P=0.423$, (E2 replication: $\beta=-0.15, z=$ $2.18, P=0.0291$, combined presentations, see Fig.2.a). If anything, there was a slight tendency towards greater confidence when choosing smaller, sooner rewards, as seen in E2, though we place low credence in this effect given it is significant only in one of the experiments under particular modeling assumptions (see supplementary materials). These results indicate that participants do not necessarily view the right decision in the intertemporal choice task as delaying gratification, casting doubt on the idea that choosing larger later rewards is the goal that people are using self-control to achieve. 


\section{Decisions are made with more confidence when participants more reliably choose options with larger time-discounted subjective values}

In line with our pre-registered hypotheses and prior work in other value-based decisionmaking domains $(28,34)$, we found in the same mixed effects logistic regression as above that “Bidding-SVD" reliably predicted MCQ choices, $\beta=5.27, z=9.33, P<0.0001$, (E2 replication: $\beta=3.95, z=7.59, P<0.0001)$. The significant slope of this logistic fit demonstrates that, on average, participants' actual MCQ choices reflect their self-reported time-discounted subjective values in the separate Bidding task. We also found that Bidding-SVD was a stronger predictor of MCQ choices when confidence was higher, $\beta=1.76, z=8.35, P<0.0001$, (E2 replication: $\beta=0.84, z=4.02, P<0.0001)$. Splitting trials into higher and lower confidence for each participant for visualization shows that when participants were highly confident that they had made the right choice ( $\geq$ median confidence), they did in fact more reliably choose whichever option had the higher time-discounted subjective value (steeper slope relating Bidding-SVD to choices when confidence was higher; see Fig.1.c). This significant BiddingSVD by confidence interaction term can also be interpreted as evidence of metacognitive insight because it shows that choices track independently estimated subjective values more closely when confidence is higher. This approach complements that of Soutschek, Tobler, and colleagues, who recently demonstrated a similar pattern using computed, rather than selfreported, subjective values $(29,30)$.

In a non-pre-registered exploratory analysis, we ran a separate model where we also entered response time and its interaction with Bidding-SVD into a mixed effects model predicting MCQ choices alongside Bidding-SVD, confidence, and their interaction. Even though confidence again interacted with Bidding-SVD to predict choice, $\beta=1.46, z=7.18, P<$ 0.0001, (E2 replication: $\beta=0.48, z=5.8, P<0.0001$ ), there was no interaction between response time and Bidding-SVD, $\beta=-0.02, \mathrm{z}=-0.2, P=0.845$, (E2 replication: $\beta=-0.13, z=-1.84, P=$ 0.0659). This finding suggests that explicit, self-reported confidence plays a metacognitive role, over and above the role of response time as an index of uncertainty $(13,34)$. 


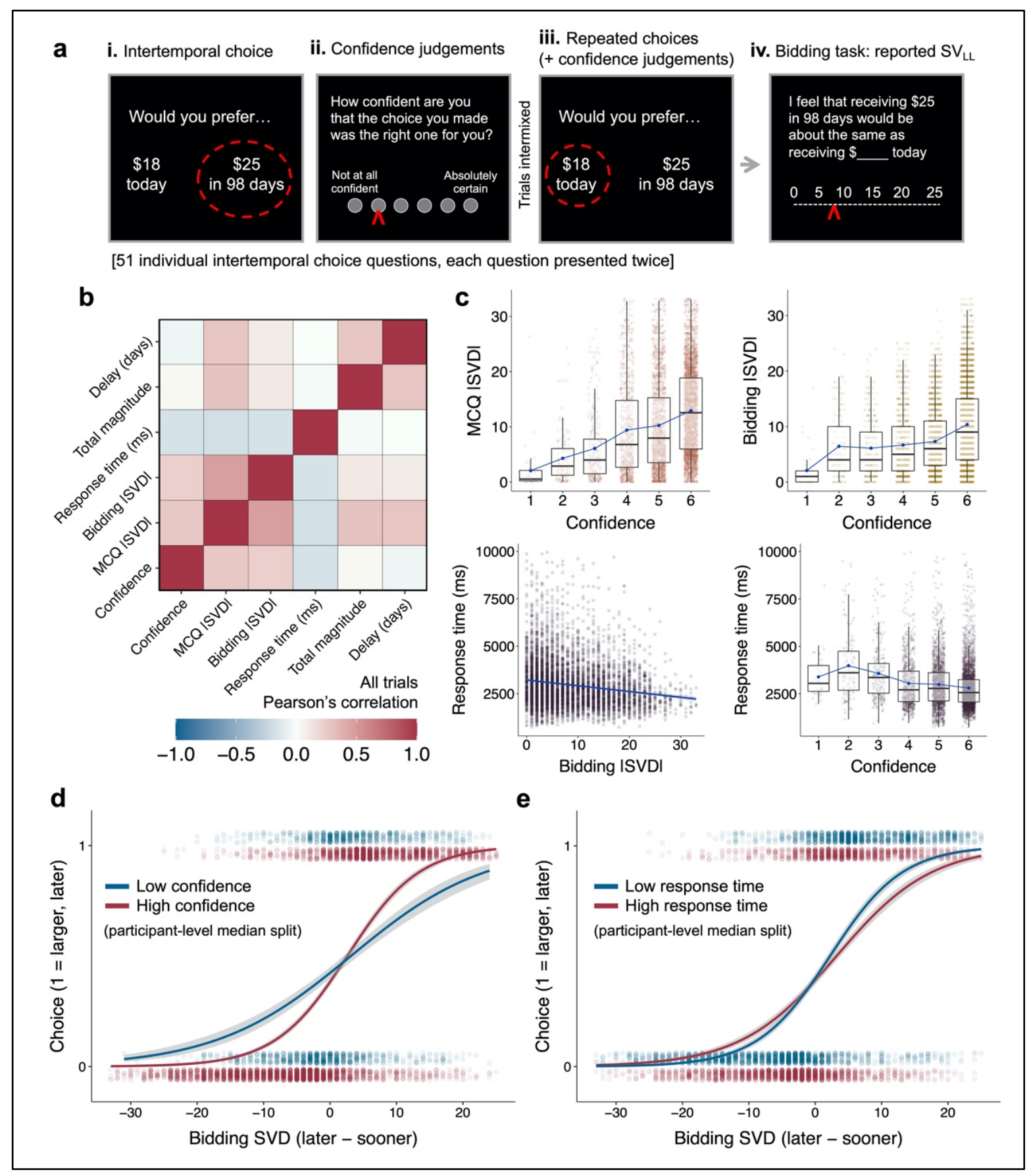

Figure 1. Correlates and consequences of confidence in intertemporal choice (E1; all independently replicated in E2, not depicted). a, Participants made intertemporal decisions (i) and judged their confidence in having made the right choice (ii). Each question appeared twice (iii). A separate "Bidding task" enabled independent estimates of time-discounted subjective value (iv). b, Correlation heatmap across all trials (combined MCQ presentations). c, Confidence was higher in decisions where the absolute subjective value difference between choice options was greater, defined either via hyperbolic discounting or Bidding task estimates. Decisions that took longer were also closer to subjective indifference and made with less confidence. d, The interaction between Bidding-SVD and confidence reveals that when participants were more confident that they had made the "right choice", they more reliably chose whichever option had higher subjective value (steeper slope predicting choice from Bidding-SVD). e, The role of response time does not survive controlling for confidence effects, suggesting a unique metacognitive role for explicit confidence. 


\section{Low confidence predicts deviations from idiosyncratic hyperbolic discounting}

In a series of pre-registered but exploratory analyses, we classified all MCQ choices in terms of whether they deviated from the idiosyncratic hyperbolic discounting function for that choice set, as a proxy for trial-by-trial choice consistency. Specifically, we identified all choices where the participant chose the reward option for which the hyperbolic model computed a lower subjective value (as per 51). This represents a deviation away from one's expected choice from the perspective of value-based decision-making, which would be the option with the higher subjective value after accounting for reward amounts and delays (Fig.2.c). Over both MCQ presentations, $7.24 \%$ of choices in E1, and 10.63\% of choices in E2 were classified as deviations. If confidence follows the same value-based decision-making principles in intertemporal choice as it does elsewhere (34), participants should be less confident when they choose an option that deviates from their overall computed preference function.

In a mixed effects logistic regression on the combined MCQ presentations data, we found evidence that decisions deviating from hyperbolic discounting were indeed made with less confidence, $\beta=-0.88, z=-7.38, P<0.0001$, (E2 replication: $\beta=-0.64, z=-9.66, P<0.0001$ ), see Fig.2.b. This was the case controlling for Bidding- $|\mathrm{SVD}|$, which also negatively predicted deviations, $\beta=-0.88, z=-5.16, P<0.0001$, (E2 replication: $\beta=-0.72, z=-5.31, P<0.0001$ ). This finding suggests that consistency with a hyperbolic discounting preference function captures aspects of decision confidence not accounted for simply by the independently estimated subjective value of choice options. Both of the above effects were significant with response time included in the model - where we found that deviation decisions were made more slowly, $\beta=0.28, z=3.68, P<0.001$, (E2 replication: $\beta=0.27, z=3.72, P<0.001)(34,48)$.

\section{Low confidence predicts changes of mind in intertemporal choices}

There were no differences in $k$ values between the two MCQ presentations in E1; presentation 1 mean $=0.0547(\mathrm{SD}=0.0850)$, presentation 2 mean=0.0582 $(\mathrm{SD}=0.0898), t(62)$ $=0.585, P=0.5605,95 \%$ CI $[-0.08,0.15]$; log-transformed. This finding is at odds with a preregistered hypothesis drawn from a self-control view that simply providing participants with the opportunity to revisit decisions would reduce delay discounting by inviting additional opportunities to override temptation, if this was indeed their goal. Instead, in a mixed effects logistic regression, we found support for another pre-registered hypothesis drawn from the value-based account that lower confidence in a decision on its first presentation would predict 
changes of mind when that question was presented again (34), $\beta=-0.76, z=-7.33, P<0.0001$, see Fig.2.d. This relationship held after controlling for Bidding- $|\mathrm{SVD}|$ and response time from the first presentation of the choice. Participants were also more likely to change their mind when options were closer together in independently estimated subjective value, Bidding-|SVD|: $\beta=-0.58, z=-5.3, P<0.0001$, and when they made their first decision more slowly, $\beta=0.29$, $z=4.02, P<0.0001$ (see 52). This analysis was performed on the data from E1 only, wherein the two presentations of the MCQ questions were identical. In E2, we designed the experiment to instead determine whether confidence would also predict changes of mind in the context of a framing manipulation. 


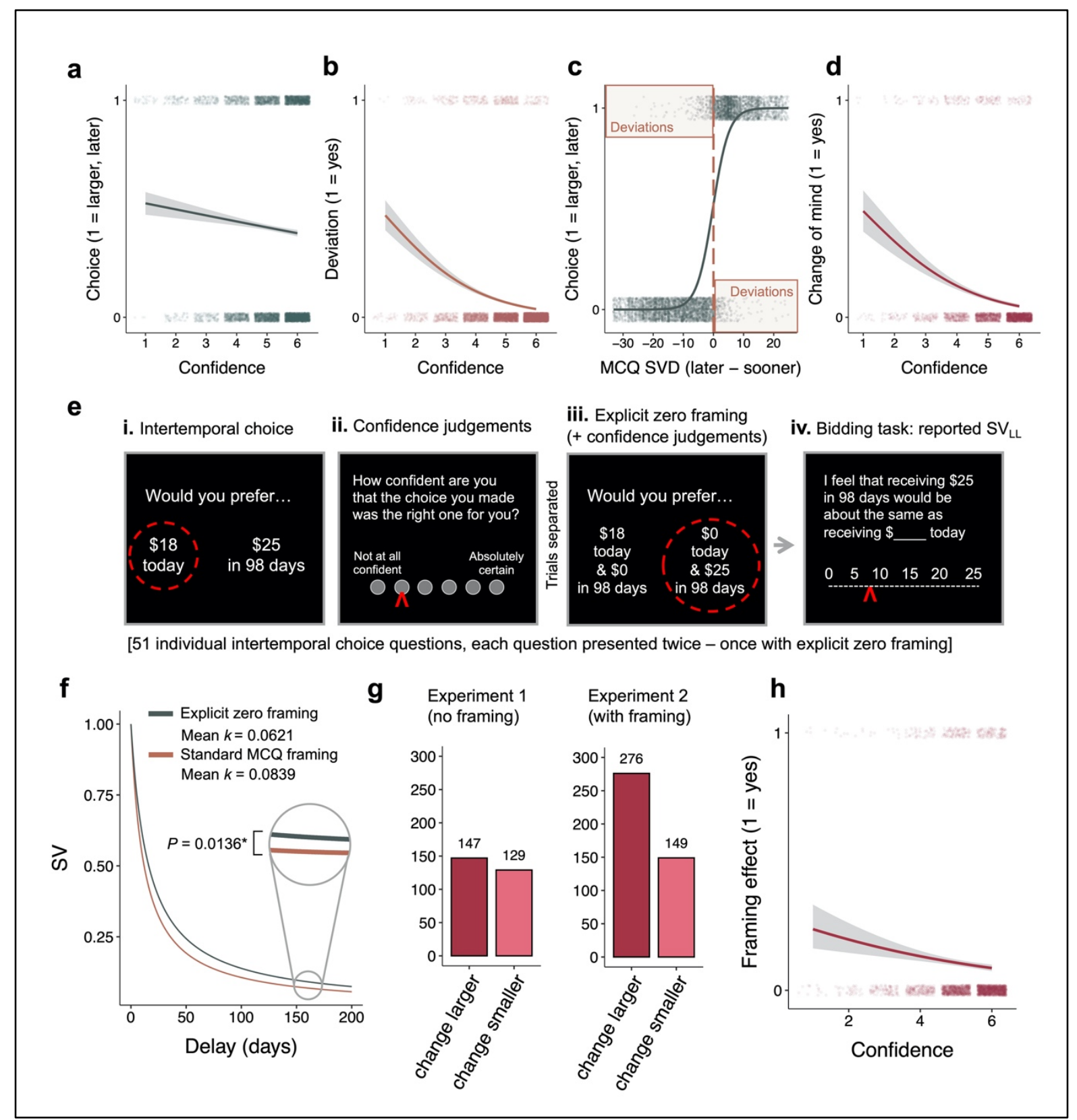

Figure 2. Confidence tracks choice inconsistency. a, Confidence does not itself predict whether participants choose to delay gratification. b, In line with a value-based account, confidence predicts choices that deviate from idiosyncratic hyperbolic discounting. c, Deviations are defined as choices of a reward with lower computed time-discounted subjective value. d, Lower initial confidence predicts a higher likelihood of changing one's mind. e, In E2, the second MCQ presentation used explicit zero framing. $\mathbf{f}$, The framing effect leads to reduced delay discounting. g, Nearly twice as many changes of mind took place in favor of the LL option between the standard phrasing and explicit zero framing presentations of the same intertemporal choice questions, in contrast to E1 where both presentations had the same phrasing and changes of mind were equally common in both directions (change of mind data for which confidence reports for the first choice were present). $\mathbf{h}$, Low confidence in an initial, standard phrasing question predicts susceptibility of that choice to the framing effect. 


\section{Low confidence predicts susceptibility to explicit zero framing}

In E2, there was a significant difference in $k$ values between the two presentations of the MCQ, which reflects the successful influence of explicit zero framing (Fig.2.e,f). Delay discounting was significantly lower in the MCQ presentation with explicit zero framing than the MCQ presentation with the standard phrasing, presentation 1 mean $=0.0839(\mathrm{SD}=0.1033)$; explicit zero presentation 2 mean $=0.0621(\mathrm{SD}=0.0951), t(53)=2.55, P=0.0136,95 \% \mathrm{CI}$ $[0.13,1.08]$, log-transformed. Almost twice as many total changes of mind were observed in favor of the larger, later, reward (276 total changes) than in favor of the smaller, sooner reward (149 total changes). This result stands in contrast to E1 where changes of mind were approximately equally common in both directions between the identically phrased MCQ presentations (see Fig.2.g.). The framing-induced shift towards LL rewards in E2 is therefore not a result of simply giving participants the opportunity to change their mind.

In support of our pre-registered hypothesis, we found evidence that lower confidence in an initial MCQ choice predicted a greater susceptibility of that choice to the explicit zero framing effect, $\beta=-0.42, z=-4.63, P<0.0001$, Fig.2.h. Once again, Bidding- $|\mathrm{SVD}|$ and response time were included in the same model as covariates, and this revealed that while Bidding-|SVD $\mid$ was a significant negative predictor of susceptibility to the framing effect, $\beta=$ $-0.36, z=-2.66, P=0.0078$, response time was not, $\beta=0.07, z=0.81, P=0.4178$. This finding indicates that self-reported confidence in a decision may have a unique role in predicting how sensitive that decision is to contextual factors, over-and-above proxies for uncertainty like response time (13). 


\section{Discussion}

When faced with an intertemporal decision, opting to delay gratification for larger, later rewards has been interpreted as a sign of self-control (2-8). Here, we elicited confidence judgements during intertemporal choice to adjudicate on this interpretation, assuming that if participants were trying to control themselves in an effort to delay gratification, they should be more confident that they had made the "right" decision when they achieved that aim. Contrary to this assumption, we found that participants were not more confident that they had made the right choice when they chose to delay gratification. Instead, participants were consistently more confident when possible rewards were: (i) farther apart in independently estimated timediscounted subjective value, (ii) less delayed into the future, and (iii) larger in total magnitude. Confidence also tracked how closely decisions aligned with the independently estimated subjective value of rewards, constituting evidence for metacognitive insight. Lower confidence predicted greater choice inconsistency quantified by deviations from hyperbolic discounting, changes of mind, and susceptibility to a patience-enhancing manipulation. Collectively, these results suggest that participants faced with intertemporal trade-offs are not necessarily attempting to delay gratification, but to maximize time-discounted subjective value regardless of whether that means choosing larger, later or smaller, sooner rewards.

Our finding that intertemporal decisions are made with greater confidence when options are farther apart in subjective value accords with a large body of research in perception (13), memory (53), and other value-based decisions (28), where confidence scales with the strength of available evidence. We also find that intertemporal choices closer to subjective indifference, as well as those made with less confidence, take longer. This finding resonates with accounts of response time as reflecting the sequential sampling of evidence towards a decision threshold or bound $(8,28,48)$. In value-based decisions, choices closer to indifference might be made with less confidence and take longer because people must sample additional internal evidence to reach a verdict $(22,54)$. This deliberation process may be particularly reliant on prospective (episodic) simulation in intertemporal decision-making, which involves anticipating the value of rewards delayed in time (for reviews see $23,24,55$ ).

The prospective simulation of decision outcomes may itself be an imperfect, noisy process, and increasingly noisy the further delayed outcomes are into the future. This conjecture has acted as an important assumption in recent modelling work where delay discounting is argued to result from internal uncertainty in future value representations $(32,33)$. We find empirical support for these ideas, in that participants were less confident in decisions 
with a more delayed larger, later option, after accounting for the subjective value difference between options. It has also been suggested recently that a higher-order metacognitive process may allocate more mental effort towards decisions that involve more available reward $(32,56)$. We also find evidence in line with this explanation. Here, participants reached greater confidence in their decisions when there was more total reward available, suggesting the allocation of additional deliberative resources towards determining the right intertemporal decision when making the right choice is deemed more important.

Although we found associations between confidence and reward magnitudes and delays, we found no relationship between confidence and the decision to delay gratification. This result strongly suggests that participants on average do not view choosing larger, later rewards in intertemporal choice tasks as their goal, calling into question the validity of treating choices of larger later rewards as indicative of a capacity for self-control. If participants are not trying to control themselves in pursuit of delaying gratification, in what sense should the choice of smaller, sooner rewards be called a failure of self-control $(57,58)$ ? Instead, our data suggest that participants faced with intertemporal choices are attempting to maximize time-discounted subjective value, and their confidence in having made the right decision reflects this goal: participants were more confident they had made the right choice when they tended to choose whichever option had the higher time-discounted subjective value, regardless of whether that was the smaller, sooner or larger, later reward.

Our interpretation should not be taken as denying any role for self-control in intertemporal decision-making. There may well be self-control related processes selectively involved for instance in choosing larger, later rewards when smaller, sooner rewards have a higher time-discounted subjective value, as argued in Figner et al., (59) or Turner et al., (8). Alternatively, self-control may be specifically associated with avoiding dynamically inconsistent preference reversals, where an initial preference for a delayed reward flips to a preference for a smaller, sooner reward simply because the two options both become closer in time. Soutschek, Tobler and colleagues have recently linked metacognitive insight in intertemporal decision-making to the willingness to adopt precommitment strategies for managing anticipated preference reversals $(29,30)$. This key finding supports a long-standing theoretical claim that people use their awareness of their own preferences to lock in highly valued alternatives because they know they may succumb to a self-control challenge as their preferences change in the future. Our study was not designed to investigate such self-control related preference reversals associated with both options becoming closer in time. Our current results do suggest, however, that simply interpreting choices of larger, later reward as a 
capacity for self-control is misguided, whether that is operationalized as choices of larger, later reward (2), a delay discounting factor such as " $k$ " (3), or some other metric such as the calculated area under a discounting curve (7).

To further test assumptions from the value-based decision-making view, we sought to determine whether confidence judgements in intertemporal choice would adhere to the same principles as they do in other value-based decision-making domains (17). Accordingly, we found that confidence predicted inconsistent decisions quantified in multiple ways. We found that participants were less confident in decisions that deviated from an idiosyncratic hyperbolic preference function, even after controlling for the independently estimated subjective value of options. This result suggests participants are aware of when a decision falls out of step with their overarching pattern of choices (see supplementary materials for further discussion). We also found that when participants reported less confidence in a decision they were more likely to change their answer when confronted with the same question again, as has been reported in non-intertemporal value-based choice (34). It remains unclear, however, whether decisions made with lower confidence are simply more labile, or whether low decision confidence acts as a learning signal for informing future decisions. This latter possibility is supported by studies showing that participants use representations of their uncertainty in other domains to learn selectively from new information (60) or to determine when to shift goals (61).

In experiment 2, confidence also predicted changes of mind in the context of a patienceenhancing framing manipulation. Decisions made with lower confidence were more susceptible to explicit zero framing, again over and above the difference in subjective value between the options. One possible mechanism by which the explicit zero framing effect works is by changing the subjective value of options, rather than by bolstering a capacity for selfcontrol per se (37). Our findings are in line with this possibility and suggest that the framing effect selectively nudges the value of options for which internal value evidence is most uncertain or ambiguous, as reflected in low decision confidence. This finding carries potential utility in applied settings where it may be possible to personalize efforts to reduce delay discounting by using decision confidence to identify the intertemporal choices most likely to benefit from intervention - circumventing the need for any model-fitting or separate estimates of subjective value.

Overall, our findings demonstrate that rather than bearing on attempts to deploy selfcontrol, confidence judgements in intertemporal choice reflect uncertainty in the noisy value estimation and comparison process by which possible outcomes are translated into actual 
decisions. These results challenge traditional self-control views and instead cast intertemporal choice as a form of value-based decision-making about future possibilities. 


\section{References}

1. Duckworth AL, Gendler TS, Gross JJ. Situational Strategies for Self-Control. Perspect Psychol Sci. 2016;11(1):35-55.

2. Logue AW. Research on self-control: An integrating framework. Behav Brain Sci. 1988;11(04):665-79.

3. Critchfield TS, Kollins SH. Temporal discounting: basic research and the analysis of socially important behavior. J Appl Behav Anal. 2001;34(1):101-22.

4. Shamosh NA, DeYoung CG, Green AE, Reis DL, Johnson MR, Conway ARA, et al. Individual differences in delay discounting: Relation to intelligence, working memory, and anterior prefrontal cortex. Psychol Sci. 2008;19(9):904-11.

5. Kirby KN. One-year temporal stability of delay-discount rates. Psychon Bull Rev. 2009;16(3):457-62.

6. Steinglass JE, Figner B, Berkowitz S, Simpson HB, Weber EU, Walsh BT. Increased capacity to delay reward in anorexia nervosa. J Int Neuropsychol Soc. 2012;18(4):773-80.

7. Jimura K, Chushak MS, Westbrook A, Braver TS. Intertemporal Decision-Making Involves Prefrontal Control Mechanisms Associated with Working Memory. Cereb Cortex. 2018;28(4):1105-16.

8. Turner BM, Rodriguez CA, Liu Q, Molloy MF, Hoogendijk M, Mcclure SM. On the neural and mechanistic bases of self-control. Cereb Cortex. 2019;29(2):732-50.

9. Yeung N, Summerfield C. Metacognition in human decision-making: Confidence and error monitoring. Philos Trans R Soc B Biol Sci. 2012;367(1594):1310-21.

10. Rouault M, McWilliams A, Allen MG, Fleming SM. Human Metacognition Across Domains: Insights from Individual Differences and Neuroimaging. Personal Neurosci. $2018 ; 1$

11. Rahnev D, Desender K, Lee ALF, Adler WT, Aguilar-Lleyda D, Akdoğan B, et al. The Confidence Database. Nat Hum Behav. 2020;1-8.

12. Fleming SM, Weil R, Nagy Z, Dolan RJ, Rees G. Relating introspective accuracy to individual differences in brain structure. Science (80- ). 2010;329:1541-3.

13. Kiani R, Corthell L, Shadlen MN. Choice certainty is informed by both evidence and decision time. Neuron. 2014;84(6):1329-42.

14. Pouget A, Drugowitsch J, Kepecs A. Confidence and certainty : distinct probabilistic quantities for different goals. Nat Neurosci. 2016;19(3):366-74. 
15. Desender K, Donner T, Verguts T. Dynamic expressions of confidence within an evidence accumulation framework. Cognition. 2021;207:104522.

16. Fleming SM, Daw ND. Self-evaluation of decision-making: A general bayesian framework for metacognitive computation. Psychol Rev. 2017;124(1):91-114.

17. Berkman ET, Hutcherson CA, Livingston JL, Kahn LE, Inzlicht M. Self-control as value-based choice. Curr Dir Psychol Sci. 2017;26(5):422-8.

18. Kable JW. Valuation, intertemporal choice, and self-control. In: Glimcher PW, Fehr E, editors. Neuroeconomics: Decision Making and the Brain: Second Edition. Academic Press; 2014. p. 173-92.

19. Rangel A, Camerer C, Montague PR. A framework for studying the neurobiology of value-based decision making. Nat Rev Neurosci. 2008;9(7):545-56.

20. Levy DJ, Glimcher PW. The root of all value: A neural common currency for choice. Curr Opin Neurobiol. 2012;22(6):1027-38.

21. Buckholtz JW. Social norms, self-control, and the value of antisocial behavior. Curr Opin Behav Sci. 2015;3:122-9.

22. Shadlen MN, Shohamy D. Decision making and sequential sampling from memory. Neuron. 2016;90(5):927-39.

23. Bulley A, Schacter DL. Deliberating trade-offs with the future. Nat Hum Behav. $2020 ; 4: 238-247$.

24. Bulley A, Henry J, Suddendorf T. Prospection and the present moment: The role of episodic foresight in intertemporal choices between immediate and delayed rewards. Rev Gen Psychol. 2016;20(1):29-47.

25. Peters J, Büchel C. Episodic future thinking reduces reward delay discounting through an enhancement of prefrontal-mediotemporal interactions. Neuron. 2010;66(1):13848.

26. Benoit RG, Gilbert SJ, Burgess PW. A neural mechanism mediating the impact of episodic prospection on farsighted decisions. J Neurosci. 2011;31(18):6771-9.

27. Peters J, Büchel C. The neural mechanisms of inter-temporal decision-making: Understanding variability. Trends Cogn Sci. 2011;15(5):227-39.

28. De Martino B, Fleming SM, Garrett N, Dolan RJ. Confidence in value-based choice. Nat Neurosci. 2013;16(1):105-10.

29. Soutschek A, Moisa M, Ruff CC, Tobler PN. Frontopolar theta oscillations link metacognition with prospective decision making. Nat Commun. 2021;12(3943):1-8.

30. Soutschek A, Tobler PN. Know your weaknesses: Sophisticated impulsiveness 
motivates voluntary self-restrictions. J Exp Psychol Learn Mem Cogn. 2020;46(9):1611-23.

31. Cooper N, Kable JW, Kim BK, Zauberman G. Brain activity in valuation regions while thinking about the future predicts individual discount rates. J Neurosci. 2013;33(32):13150-6.

32. Gershman S, Bhui R. Rationally inattentive intertemporal choice. Nat Commun. 2020;11(3365):1-8.

33. Gabaix X, Laibson D. Myopia and discounting. NBER Work Pap Ser. 2017;

34. Folke T, Jacobsen C, Fleming SM, De Martino B. Explicit representation of confidence informs future value-based decisions. Nat Hum Behav. 2016;1:1-8.

35. Lempert KM, Phelps EA. The malleability of intertemporal choice. Trends Cogn Sci. 2016;20(1):64-74.

36. Jenkins AC, Hsu M. Dissociable contributions of imagination and willpower to the malleability of human patience. Psychol Sci. 2017;28(7):894-906.

37. Magen E, Kim B, Dweck CS, Gross JJ, McClure SM. Behavioral and neural correlates of increased self-control in the absence of increased willpower. Proc Natl Acad Sci. 2014;111(27):9786-91.

38. Lempert KM, Mechanic-hamilton DJ, Xie L, Wisse LEM, Flores R De, Wang J, et al. Neural and behavioral correlates of declarative memory are associated with temporal discounting in older adults. Neuropsychologia. 2020;146:1-49.

39. Yu LQ, Lee S, Katchmar N, Satterthwaite TD, Kable JW, Wolf DH. Steeper discounting of delayed rewards in schizophrenia but not first-degree relatives. Psychiatry Res. 2017;252:303-9.

40. Mazur JE. An adjusting procedure for studying delayed reinforcement. In: Commons ML, Mazur JE, Nevin JA, Rachlin H, editors. Quantitative analyses of behavior, Vol 5 The effect of delay and of intervening events on reinforcement value. NJ: Lawrence Erlbaum Associates, Inc; 1987. p. 55-73.

41. Odum AL. Delay Discounting: I'm a k, You're a k. J Exp Anal Behav. 2011;96(3):427-39.

42. Lempert KM, MacNear KA, Wolk DA, Kable JW. Links between autobiographical memory richness and temporal discounting in older adults. Sci Rep. 2020;10:6431.

43. Team RC. R: A language and environment for statistical computing. R Foundation for Statistical Computing. Vienna, Austria; 2019.

44. Wickham H, Averick M, Bryan J, Chang W, McGowan L, François R, et al. Welcome 
to the Tidyverse. J Open Source Softw. 2019;4(43):1686.

45. Bates D, Mächler M, Bolker B, Walker S. Fitting Linear Mixed-Effects Models Using lme4. J Stat Softw. 2015;67(1):1-48.

46. Kuznetsova A, Brockhoff PB, Christensen RHB. lmerTest Package: Tests in Linear Mixed Effects Models. J Stat Softw. 2017;82(13).

47. Konovalov A, Krajbich I. Revealed strength of preference: Inference from response times. Judgm Decis Mak. 2019;14(4):381-94.

48. Rodriguez CA, Turner BM, McClure SM. Intertemporal choice as discounted value accumulation. PLoS One. 2014;9(2).

49. Dai J, Busemeyer JR. A probabilistic, dynamic, and attribute-wise model of intertemporal choice. J Exp Psychol Gen. 2014;143(4):1489-514.

50. Bürkner P-C, Vuorre M. Ordinal Regression Models in Psychology: A Tutorial. Adv Methods Pract Psychol Sci. 2019;2(1):77-101.

51. Lempert KM, Lackovic SF, Tobe RH, Glimcher PW, Phelps EA. Propranolol reduces reference-dependence in intertemporal choice. Soc Cogn Affect Neurosci. 2017;12(9):1394-401.

52. Alós-Ferrer C, Garagnani M. Choice consistency and strength of preference. Econ Lett. 2021;198:109672.

53. Koriat A. Monitoring One's Own Knowledge during Study: A Cue-Utilization Approach to Judgments of Learning. J Exp Psychol Gen. 1997;126(4):349-70.

54. Bakkour A, Palombo DJ, Zylberberg A, Kang YH, Reid A, Verfaellie M, et al. The hippocampus supports deliberation during value-based decisions. Elife. 2019;8:1-28.

55. Schacter DL, Benoit RG, Szpunar KK. Episodic future thinking: mechanisms and functions. Curr Opin Behav Sci. 2017;17:41-50.

56. Ballard IC, Kim B, Liatsis A, Aydogan G, Cohen JD, McClure SM. More Is meaningful: The magnitude effect in intertemporal choice depends on self-control. Psychol Sci. 2017;28(10):1443-54.

57. Vosgerau J, Scopelliti I, Huh YE. Exerting self-ccontrol $\neq$ sacrificing pleasure. J Consum Psychol. 2020;30(1):181-200.

58. Lempert KM, Steinglass JE, Pinto A, Kable JW, Simpson HB. Can delay discounting deliver on the promise of RDoC? Psychol Med. 2019;49(2):190-9.

59. Figner B, Knoch D, Johnson EJ, Krosch AR, Lisanby SH, Fehr E, et al. Lateral prefrontal cortex and self-control in intertemporal choice. Nat Neurosci. 2010;13(5):538-9. 
60. Meyniel F, Dehaene S. Brain networks for confidence weighting and hierarchical inference during probabilistic learning. Proc Natl Acad Sci U S A. 2017;114(19):E3859-68.

61. Carlebach N, Yeung N. Subjective Confidence Acts as an Internal Cost-Benefit Factor When Choosing Between Tasks. J Exp Psychol Hum Percept Perform. 2020;46(7):729-48.

\section{Funding information}

A.B. is supported by an Australian National Health and Medical Research Council CJ Martin Biomedical Fellowship APP1162811 (GNT1162811), and an Australian Research Council Discovery Project Grant DP210101572 . K.M.L was supported by a National Research Service Award from the National Institute on Aging F32-AG-054032-02. M.I. is supported by an Australian Research Council Future Fellowship Grant FT160100096 and an ARC Discovery Project Grant DP180101548. DLS is supported by by National Institute of Mental Health grant R01 MH060941 and National Institute on Aging grant R01 AG008441. 